Revista de Matemática: Teoría y Aplicaciones 2006 13(1) : 1-15

CIMPA - UCR - CCSS ISSN: 1409-2433

\title{
GENERADORES DE NÚMEROS PRIMOS Y FACTORIZADORES DE NÚMEROS COMPUESTOS
}

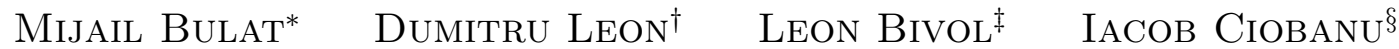 \\ Aurel ZGureanu
}

Recibido/Received: 21 Oct 2005 - Aceptado/Accepted: 25 Aug 2006

\begin{abstract}
Resumen
La serie de los números naturales se representa por una matriz multidimensional. En la base de las propiedades de estas matrices se examinan los problemas:

a) la distribución de los números primos en las matrices multidimensionales,

b) la factorización de los números compuestos.

Para resolver el problema de la distribución, se elaboró un generador de números primos en el lenguaje Delphi. Este programa sirve también como generador de números compuestos de Mersenne. Al generar un número compuesto de Mersenne automáticamente se encuentra y uno de sus divisores.

El problema de la factorización en el lenguaje Delphi se resuelve para los números de la forma $2^{i}-1$, donde $i$ es un número natural. En particular, para $i$ primo se factorizan los números compuestos de Mersenne.
\end{abstract}

Palabras clave: matrices multidimensionales, divisores primos, números de Mersenne.

\footnotetext{
${ }^{*}$ Departamento de Matemáticas, Academia de Transportes, Informática y Comunicaciones; Chisinau, Moldavia. E-Mail: msbulat@mail.ru

${ }^{\dagger}$ MOBIASBANC, Chisinau, Moldavia. E-Mail: diLeon@yandex.ru

${ }^{\ddagger}$ Departamento de Matemáticas e Informática de la Academia de Transportes, Informática y Comunicaciones (ATIC), Chisinau, Moldavia. E-Mail: cirese2006@yahoo.com

${ }^{\S}$ Departamento de Matemáticas e Informática de la Academia de Transportes, Informática y Comunicaciones(ATIC), Chisinau, Moldavia. E-Mail: ciobanu@mail.ru

`Departamento de Matemáticas e Informática de la Academia de Transportes, Informática y Comunicaciones(ATIC), Chisinau, Moldavia.E-Mail: aurelzgureanu@rambler.ru
} 


\begin{abstract}
The sequence of natural numbers presents itself as a multidimensional array. Two problems are solved on the basis of these arrays:

a) distribution of prime numbers in multidimensional array,

b) factoring of composite numbers.

For solving the problems related to the distribution of prime numbers, there was developed a generator of prime numbers in the Delphi programming language. The program serves as well as a generator of Mersenne composite numbers. While generating a Mersenne composite number, one of its divisors is automatically defined.

The problem of factoring in Delphi is solved for numbers of the form $2^{i}-1$, where $i$ is a natural number. Mersenne composite numbers are factoring in particular for the prime $i$.
\end{abstract}

Keywords: multidimensional array, divisor prime, Mersenne numbers.

Mathematics Subject Classification: 05C60, 68R10.

\title{
1 Introducción
}

Para resolver problemas en sistemas de grandes dimensiones, en [1] y [2] fue introducida la transformación $\Phi(\vec{R})$. Por medio de esta transformación, los sistemas se representan por matrices multidimensionales. En [3] se examinaron unas aplicaciones de tales matrices. Una de estas aplicaciones es la representación de la serie de los números naturales por matrices multidimensionales. Tal representación permite desde otro punto de vista, resolver el problema de la distribución de los números primos en la serie de los números naturales [4]. Actualmente, debido al amplio desarrollo de las tecnologías de información, adquiere cada vez mayor importancia el problema de la protección de la información. Los números primos juegan un papel principal en la resolución de este problema. En sistemas de grandes dimensiones [1], es imposible resolver problemas sin aplicación de la computadora. Las propiedades de las matrices multidimensionales permiten elaborar generadores de números primos y factorizadores para números compuestos. En este trabajo se han examinado unas propiedades adicionales de las matrices multidimensionales. En la base de estas propiedades, junto con otras propiedades, se han elaborado programas en el lenguaje Delphi.

\section{Matrices multidimensionales}

Sea $n$ conjuntos $X_{1}=\left\{x_{11}, \ldots, x_{1 m_{1}}\right\}, X_{2}=\left\{x_{21}, \ldots, x_{2 m_{2}}\right\}, \ldots, X_{n}=\left\{x_{n_{1}}, \ldots, x_{n m_{n}}\right\}$ sobre los cuales, por medio de los elementos del conjunto $\Omega=\left\{\omega_{1}, \ldots, \omega_{t}\right\}$ están definidas $r$ relaciones $R_{X_{i_{1}} X_{i_{2}} \ldots X_{i_{d}}}$ donde $2 \leq d \leq n$ y $i_{j} \in\{1, \ldots, n\}$ para cualquier $j \in\{1, \ldots, d\}$. Estas relaciones forman el conjunto $R_{1}$. Al ordenar los elementos de este conjunto $[1,3]$ obtendremos el vector $\vec{R}_{1}$. Según [1,3] por medio de la transformación $\Phi\left(\vec{R}_{1}\right)$ construimos la matriz $n$-dimensional $A=\Phi\left(\vec{R}_{1}\right)$. En [3] se demostró que los elementos de esta matriz 
representan números enteros no negativos en la base $t$. Pasamos a la base $10 \mathrm{y}$ escribimos esta matriz en la forma de una matriz bidimensional

$$
A=\left[\begin{array}{cccc}
a_{11 \ldots 1} & a_{11 \ldots 2} & \ldots & a_{1 m_{2} \ldots m_{n}} \\
a_{21 \ldots 1} & a_{21 \ldots 2} & \ldots & a_{2 m_{2} \ldots m_{n}} \\
\ldots & \ldots & \ldots & \ldots \\
a_{m_{1} 1 \ldots 1} & a_{m_{1} 1 \ldots 2} & \ldots & a_{m_{1} m_{2} \ldots m_{n}}
\end{array}\right]
$$

Consideremos un caso particular con las condiciones:

$$
\begin{gathered}
\Omega=\{0,1,2, \ldots, m-1\}, r=n, d=2 \\
m_{1}=m_{2}=\ldots=m_{n}=t=m \\
R_{1}=\left(R_{X_{1} X_{2}}, R_{X_{2} X_{3}}, \ldots, R_{X_{n-1} X_{n}}, R_{X_{n} X_{1}}\right) \\
R_{X_{1} X_{2}}=R_{X_{2} X_{3}}=\ldots=R_{X_{n-1} X_{n}}=R_{X_{n} X_{1}} \\
\forall i, j \quad R_{X_{i} X_{j}}=\left[\begin{array}{ccccc}
0 & 1 & 2 & \ldots & m-1 \\
0 & 1 & 2 & \ldots & m-1 \\
\ldots & \ldots & \ldots & \ldots & \ldots \\
0 & 1 & 2 & \ldots & m-1
\end{array}\right]
\end{gathered}
$$

Con estas condiciones la matriz $n$-dimensional (1) tiene una forma específica

$$
A=\begin{gathered}
K_{0} \\
K_{1} \\
K_{2} \\
\vdots \\
K_{m-1}
\end{gathered}\left[\begin{array}{ccccc}
0 & m+0 & 2 m & \ldots & m^{n}-(m-0) \\
1 & m+1 & 2 m+1 & \ldots & m^{n}-(m-1) \\
2 & m+2 & 2 m+2 & \ldots & m^{n}-(m-2) \\
\vdots & \vdots & \vdots & \ddots & \vdots \\
m-1 & m+(m-1) & 2 m+(m-1) & \ldots & m^{n}-1
\end{array}\right]
$$

Denotemos las líneas de esta matriz por $K_{0}, K_{1}, \ldots, K_{m-1}$. Para $m=6$ y $n=3$ tenemos:

$$
\begin{aligned}
& K_{0}\left[\begin{array}{llllllllllllllllllll}
0 & 6 & 12 & 18 & 24 & 30 & 36 & 42 & 48 & 54 & 60 & 66 & 72 & 78 & 84 & 90 & 96 & 102 & \ldots & 210
\end{array}\right] \\
& \left.\begin{array}{lllllllllllllllllllll}
K_{1} & 1 & \mathbf{7} & \mathbf{1 3} & \mathbf{1 9} & 25 & \mathbf{3 1} & \mathbf{3 7} & \mathbf{4 3} & 49 & 55 & \mathbf{6 1} & \mathbf{6 7} & \mathbf{7 3} & \mathbf{7 9} & 85 & 91 & \mathbf{9 7} & \mathbf{1 0 3} & \ldots & \mathbf{2 1 1}
\end{array}\right] \\
& A=K_{2} \quad \begin{array}{llllllllllllllllllllll}
\mathbf{2} & 8 & 14 & 20 & 26 & 32 & 38 & 44 & 50 & 56 & 62 & 68 & 74 & 80 & 86 & 92 & 98 & 104 & \ldots & 212
\end{array} \\
& \begin{array}{lllllllllllllllllllll}
K_{3} & 3 & 9 & 15 & 21 & 27 & 33 & 39 & 45 & 51 & 57 & 63 & 69 & 75 & 81 & 87 & 93 & 99 & 105 & \ldots & 213
\end{array} \\
& \begin{array}{llllllllllllllllllllll}
K_{4} & 4 & 10 & 16 & 22 & 28 & 34 & 40 & 46 & 52 & 58 & 64 & 70 & 76 & 82 & 88 & 94 & 100 & 106 & \ldots & 214 \\
\hline
\end{array}
\end{aligned}
$$

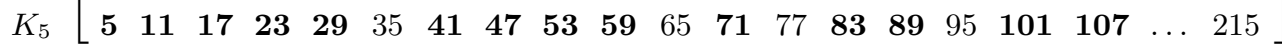

Veamos unas propiedades de las matrices (7) [4]:

1. Las líneas $K_{i}$ representan progresiones aritméticas con la razón $m$ y con el primer término igual a $i$. Las columnas también representan progresiones aritméticas con la razón igual a 1. 
2. Si $x \in K_{i}$ e $y \in K_{j}$ entonces $x \cdot y \in K_{(i \cdot j)(\bmod m)} \mathrm{y} x+y \in K_{(i+j)(\bmod m)}$.

3. Si $(i, m)=1$ ( $i$ y $m$ son recíprocamente primos), entonces la línea $K_{i}$ contiene un número infinito de números primos (para $n=\infty$ ).

4. Para $(i, m) \neq 1$ e $i$ compuesto, la línea $K_{i}$ no contiene números primos; para $i$ primo e $(i, m) \neq 1$ la línea $K_{i}$ contiene solamente un número primo, esto es el número $i$.

Veamos la matriz (8). Ya que $(1,6)=1$ y $(5,6)=1$ entonces en las líneas $K_{1}$ y $K_{5}$ la cantidad de los números primos crece infinitamente cuando $n \rightarrow \infty$ (el número de líneas se queda constante). Luego, $(2,6)=2,(3,6)=3$. Ya que 2 y 3 son primos, entonces cada una de las líneas $K_{2}$ y $K_{3}$ contiene solamente un número primo, 2 y 3 respectivamente. En $K_{0}$ todos los números son compuestos.

De las últimas dos propiedades resulta que para $m$ primo, todas las líneas de la matriz contienen números primos. La línea $K_{0}$ contiene solamente un número primo (el número $m$ ). Las demás líneas contienen un número infinito de números primos.

En la matriz (8) los números primos están escritos con caracteres gruesos.

5. El número natural $N$ pertenece a la línea $K_{j}$ si y solo si $m$ es un divisor del número $N-j$, es decir si y solo si se cumple la condición

$$
(N-j) \vdots m
$$

6. La estructura de la matriz es invariante bajo los parámetros $m$ y $n$, es decir, al cambiar $m$ y $n$, la matriz queda compuesta de progresiones aritméticas indicadas en $1)$.

7. El número de líneas de la matriz (7) que contienen un número infinito de números primos, es igual a la función de Euler $\varphi(m)[4,5]$. Si $(i, m)=1$ entonces $K_{i}$ contiene un número infinito de números primos. Las propiedades de las matrices multidimensionales, nos permiten estimar el porcentaje de los números primos en la serie de los números naturales. En [7] se obtuvo la estimación igual a 26.66\% (http://bajandin.narod.ru). Consideremos que todos los elementos de la línea $K_{i}$ son primos. Ya que la matriz tiene $m$ líneas, entonces la cota superior del porcentaje indicado es igual a $\frac{\varphi(m)}{m} \cdot 100 \% \forall n$. Calculemos la cota para distintos valores de $m$. Sea $m=p_{1} \cdot p_{2} \cdot \ldots \cdot p_{k}$, donde $p_{i}$ son números primos. En este caso $\varphi(m)=$ $\left(p_{1}-1\right) \cdot\left(p_{2}-1\right) \cdot \ldots \cdot\left(p_{k}-1\right) \mathrm{y}$, por eso $\frac{\varphi(m)}{m}=\frac{\left(p_{1}-1\right) \cdot\left(p_{2}-1\right) \cdot \ldots \cdot\left(p_{k}-1\right)}{p_{1} \cdot p_{2} \cdot \ldots \cdot p_{k}}$.

Sea $m=p_{1}^{\lambda_{1}} \cdot p_{2}^{\lambda_{2}} \cdot \ldots \cdot p_{k}^{\lambda_{k}}$. Ya que en este caso $\varphi(m)=p_{1}^{\lambda_{1}-1} \cdot\left(p_{1}-1\right) \cdot p_{2}^{\lambda_{2}-1} \cdot\left(p_{2}-\right.$ 1) $\cdot \ldots \cdot p_{k}^{\lambda_{k}-1}\left(p_{k}-1\right)$ entonces

$$
\frac{\varphi(m)}{m}=\frac{p_{1}^{\lambda_{1}-1} \cdot\left(p_{1}-1\right) \cdot p_{2}^{\lambda_{2}-1} \cdot\left(p_{2}-1\right) \cdot \ldots \cdot p_{k}^{\lambda_{k}-1}\left(p_{k}-1\right)}{p_{1}^{\lambda_{1}} \cdot p_{2}^{\lambda_{2}} \cdot \ldots \cdot p_{k}^{\lambda_{k}}}
$$




$$
\begin{aligned}
& =\frac{p_{1}^{\lambda_{1}}\left(1-\frac{1}{p_{1}}\right) \cdot p_{2}^{\lambda_{2}}\left(1-\frac{1}{p_{2}}\right) \cdot \ldots \cdot p_{k}^{\lambda_{k}}\left(1-\frac{1}{p_{k}}\right)}{p_{1}^{\lambda_{1}} \cdot p_{2}^{\lambda_{2}} \cdot \ldots \cdot p_{k}^{\lambda_{k}}} \\
& =\frac{\left(p_{1}-1\right) \cdot\left(p_{2}-1\right) \cdot \ldots \cdot\left(p_{k}-1\right)}{p_{1} \cdot p_{2} \cdot \ldots \cdot p_{k}} .
\end{aligned}
$$

Así pues, la cota depende solamente de los divisores simples de $m$. Por eso, es suficiente tomar $m=p_{1} \cdot p_{2} \cdot \ldots \cdot p_{k}$. Al tomar, por ejemplo,

$m=2 \cdot 3 \cdot 5 \cdot 7 \cdot 11 \cdot 13 \cdot 17 \cdot 19 \cdot 23 \cdot 29 \cdot 31 \cdot 37 \cdot 41 \cdot 43 \cdot 47 \cdot 53 \cdot 59 \cdot 61 \cdot 67 \cdot 71 \cdot 73 \cdot 79 \cdot 83 \cdot 89$

obtendremos la cota superior del porcentaje de los números primos en la serie de los números naturales igual a $12,16 \%$.

Veamos otras propiedades de la matriz en dependencia de otros parámetros y de otras condiciones. Supongamos que en (2) tenemos la condición $r>n$ y consideremos el vector $\vec{R}_{1}^{*}=\left(R_{1,2}, R_{2,3}, \ldots, R_{n, 1}, R\left(s_{1}\right), \ldots, R\left(s_{k}\right)\right)$, donde $\forall i, j \quad R_{i, j}=R_{x_{i}, x_{j}}$ y $R\left(s_{i}\right)$ es la matriz que se obtiene de la matriz (6) por la sustitución de cada uno de sus elementos por el elemento $s_{i} \in\{0,1, \ldots, m-1\}$, es decir

$$
R\left(s_{i}\right)=\left[\begin{array}{lll}
s_{i} & \ldots & s_{i} \\
& \ddots & \\
s_{i} & \ldots & s_{i}
\end{array}\right]
$$

Ya que todos los elementos de esta matriz son iguales entre si, entonces la columna $R\left(s_{i}\right)$ contiene el mismo número $s_{i}$ en todas las líneas [3].

Sea, $A=\Phi\left(\vec{R}_{1}\right), A^{*}=\Phi\left(\vec{R}_{1}^{*}\right)$ y $a_{j_{1} \ldots j_{n}}$-un elemento arbitrario de la matriz $A$. Al aumentar $r$, según la definición de la transformación $\Phi\left(\vec{R}_{1}\right)[1,2]$, el número de elementos de la matriz no cambia. Pueden cambiar solamente los elementos de la matriz. Por eso podemos designar por $a_{j_{1} \ldots j_{n}}^{*}$ el elemento arbitrario de la matriz $A^{*}$.

Teorema 1 Sea $\vec{R}_{1}=\left(R_{1,2}, R_{2,3}, \ldots, R_{n, 1}\right), \quad \vec{R}_{1}^{*}=\left(R_{1,2}, R_{2,3}, \ldots, R_{n, 1}, R\left(s_{1}\right) \ldots\right.$, $\left.R\left(s_{k}\right)\right), A=\Phi\left(\vec{R}_{1}\right)$ y $A^{*}=\Phi\left(\vec{R}_{1}^{*}\right)$ entonces

$$
a_{j_{1} \ldots j_{n}}^{*}=a_{j_{1} \ldots j_{n}} \cdot m^{k}+s
$$

$$
\text { donde } \quad s=s_{1} m^{k-1}+s_{2} m^{k-2}+\ldots+s_{k} .
$$

Demostración: Los elementos de $A$ y $A^{*}$ son números enteros no negativos en la base $m$. Supongamos que $a_{j_{1} \ldots j_{n}}=\sigma_{1} \sigma_{2} \ldots \sigma_{n}$, donde $\sigma_{i} \in\{0,1,2, \ldots, m-1\}$. Entonces podemos escribir $a_{j_{1} \ldots j_{n}}=\sigma_{1} m^{n-1}+\sigma_{2} m^{n-2}+\ldots+\sigma_{n} \quad$ y $\quad a_{j_{1} \ldots j_{n}}^{*}=\sigma_{1} m^{n+k-1}+\sigma_{2} m^{n+k-2}+$ $\ldots+\sigma_{n} m^{k}+s_{1} m^{k-1}+s_{2} m^{k-2}+\ldots+s_{k}=m^{k}\left(\sigma_{1} m^{n-1}+\sigma_{2} m^{n-2}+\ldots+\sigma_{n}\right)+s_{1} m^{k-1}+$ $s_{2} m^{k-2}+\ldots+s_{k}=a_{j_{1} \ldots j_{n}} \cdot m^{k}+s$, donde $s=s_{1} m^{k-1}+s_{2} m^{k-2}+\ldots+s_{k}$, lo que se trataba de demostrar. 
Corolario 1 Las columnas y las líneas de la matriz $A^{*}$ son progresiones aritméticas con las razones $m^{k}$ y $m^{k+1}$ respectivamente.

En efecto, según la estructura de la matriz (1) para líneas, tenemos: $a_{\left(j_{1}+1\right) j_{2} \ldots j_{n}}^{*}-$ $a_{j_{1} j_{2} \ldots j_{n}}^{*}=\left(a_{\left(j_{1}+1\right) j_{2} \ldots j_{n}} \cdot m^{k}+s\right)-\left(a_{j_{1} j_{2} \ldots j_{n}} \cdot m^{k}+s\right)=m^{k}\left(a_{\left(j_{1}+1\right) j_{2} \ldots j_{n}}-a_{j_{1} j_{2} \ldots j_{n}}\right)=m^{k}$.

Para columnas se obtiene: $a_{j_{1} j_{2} \ldots\left(j_{n}+1\right)}^{*}-a_{j_{1} j_{2} \ldots j_{n}}^{*}=\left(a_{j_{1} j_{2} \ldots\left(j_{n}+1\right)} \cdot m^{k}+s\right)-\left(a_{j_{1} j_{2} \ldots j_{n}}\right.$. $\left.m^{k}+s\right)=m^{k}\left(a_{j_{1} j_{2} \ldots\left(j_{n}+1\right)}-a_{j_{1} j_{2} \ldots j_{n}}\right)=m^{k} \cdot m=m^{k+1}$.

Corolario 2 Si $s \in K_{i}$ de la matriz n-dimensional $A$ con las condiciones (2)-(6) entonces todos los elementos $a_{j_{1} j_{2} \ldots j_{n}}^{*}$ de la matriz $A^{*}$ pertenecen a $K_{i}$ de la matriz $(n+k)$ dimensional con las mismas condiciones (2)-(6) en las cuales $n$ está sustituido por $n+k$.

En efecto. Supongamos que $s$ es un elemento de la matriz $A$ y $s \in K_{i}$. Ya que $m \in K_{0}$ entonces, conforme la propiedad (2), para cualquier $a_{j_{1} \ldots j_{n}}$ se cumple la condición $\left(a_{j_{1} \ldots j_{n}} \cdot m^{k}+s\right) \in K_{i}$, es decir $a_{j_{1} \ldots j_{n}}^{*} \in K_{i}$.

De este corolario resulta que si $s$ pertenece a una línea que no contiene números primos, entonces la matriz $A^{*}$ está conformada solamente por números compuestos. La matriz $A^{*}$ puede tener números primos solamente en el caso cuando $s \in K_{i} \quad$ y $\quad(i, m)=1$.

Hace falta señalar que si $s \in K_{i}$, entonces $\left(a_{j_{1} \ldots j_{n}} \cdot m^{k}+s\right) \in K_{i}$ para cualquier $k$. Por eso, para obtener intervalos de números con algunas propiedades de la línea $K_{i}$, podemos tomar valores arbitrarios de $k$.

Teorema 2 Sea $\vec{R}_{1}=\left(R_{1,2}, R_{2,3}, \ldots, R_{n, 1}\right), \quad \vec{R}_{1}^{*}=\left(R\left(s_{1}\right), \ldots, R\left(s_{k}\right), R_{1,2}, R_{2,3}, \ldots\right.$, $\left.R_{n, 1}\right), \quad A=\Phi\left(\vec{R}_{1}\right) \quad$ y $\quad A^{*}=\Phi\left(\vec{R}_{1}^{*}\right)$ entonces

$$
a_{j_{1} \ldots j_{n}}^{*}=a_{j_{1} \ldots j_{n}}+s \cdot m^{k}
$$

donde $s=s_{1} m^{k-1}+s_{2} m^{k-2}+\ldots+s_{k}$.

Demostración: Supongamos que $a_{j_{1} \ldots j_{n}}=\sigma_{1} \sigma_{2} \ldots \sigma_{n}$, donde $\sigma_{i} \in\{1,2, \ldots, m-1\}$. Entonces, según la transformación $\Phi$ tenemos: $a_{j_{1} \ldots j_{n}}^{*}=s_{1} s_{2} \ldots s_{k} \sigma_{1} \sigma_{2} \ldots \sigma_{n}=s_{1} m^{k+n-1}+$ $\ldots+s_{k} m^{n}+\sigma_{1} m^{n-1}+\ldots+\sigma_{n}=m^{n}\left(s_{1} m^{k-1}+\ldots+s_{k}\right)+\sigma_{1} m^{n-1}+\ldots+\sigma_{n}=a_{j_{1} \ldots j_{n}}+s \cdot m^{n}$, donde $s=s_{1} m^{k-1}+s_{2} m^{k-2}+\ldots+s_{k}$, lo que se trataba de demostrar.

Corolario 3 Las columnas y las líneas de la matriz $A^{*}$ son progresiones aritméticas con las razones 1 y $m$ respectivamente.

Corolario 4 Si $a_{j_{1} \ldots j_{n}} \in K_{i}$ entonces $a_{j_{1} \ldots j_{n}}^{*} \in K_{i}$.

En efecto, $m \in K_{0} \Rightarrow s \cdot m^{n} \in K_{0}$. Ya que $a_{j_{1} \ldots j_{n}} \in K_{i}$ entonces según la propiedad 2) $a_{j_{1} \ldots j_{n}}+s \cdot m^{n} \in K_{i}$ es $\operatorname{decir} a_{j_{1} \ldots j_{n}}^{*} \in K_{i}$. 


\section{Generadores de números primos}

Se sabe $[5,6]$, que si las condiciones

$$
a^{i} \equiv 1(\bmod N), \quad(a, N)=1
$$

se cumplen para $i=N-1$ y no se cumplen $\forall i<(N-1)$, entonces $N$ es número primo.

En la base de las propiedades de las matrices multidimensionales y de las condiciones (14), se elaboró un generador de números primos en el lenguaje Delphi (ver la Fig. 1, las inscripciones están hechas en rumano). El generador funciona con las restricciones:

$$
7 \leq N \leq 2200000000, \quad a \cdot(N-1) \leq 2200000000, \quad 2 \leq i \leq N-1 .
$$

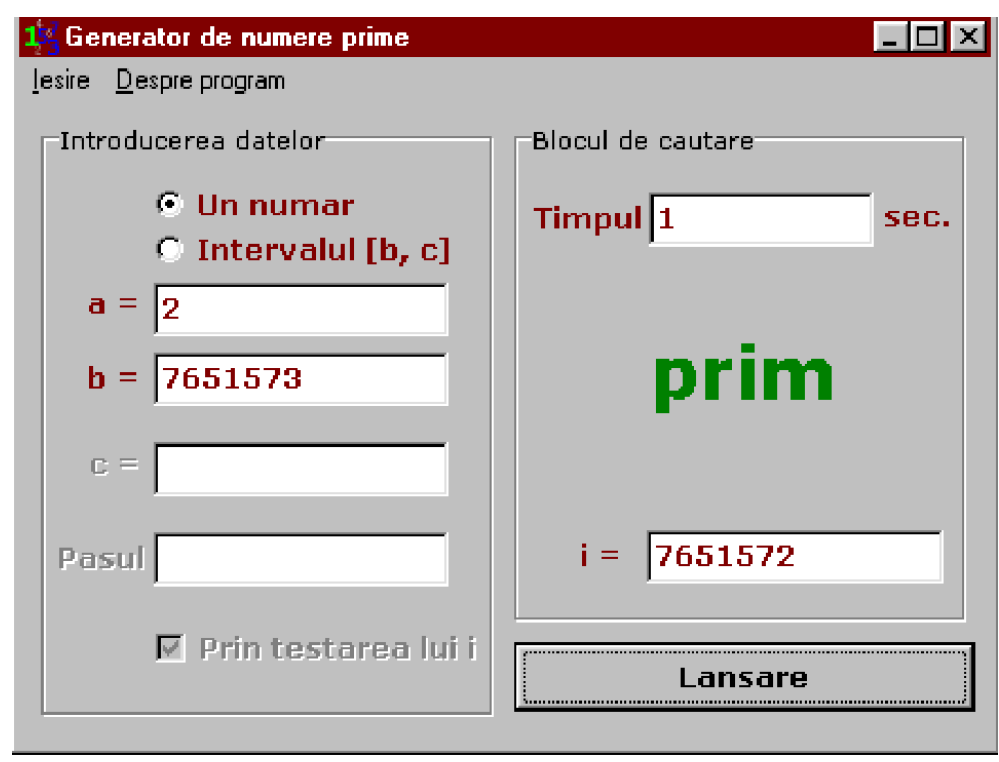

Figura 1: Generador de números primos en lenguaje Delphi, caso $N=7651573$.

$\mathrm{Al}$ círculo "un numar" lo activamos (hacemos click) en el caso cuando investigamos solamente un número. En la celda "a" escribimos el valor de $a$ de las condiciones (14). El número $N$ que se investiga se escribe en la celda "b". Al activar el botón "Lansare", en la pantalla aparece el resultado de la investigación: "Prim"(Primo) o "Compus"(Compuesto). En la celda "i" aparece el valor de $i$ de las condiciones (14). En la Fig.1 se examinó el número 7651573. Ya que $i=N-1$, entonces el número es primo. En la celda "timpul" aparece la duración de la investigación. En la Fig.2 tenemos el caso cuando el número es compuesto. El resultado "Compus" aparece cuando $i$ no es divisor de $N-1$ o no existe. Este resultado es definitivo. Si $i$ existe, es menor que $N-1$ y es divisor de $N-1$, entonces en la pantalla aparece "Primo". Este resultado no es definitivo. En este caso hay que repetir la investigación con otro valor de $a$. 


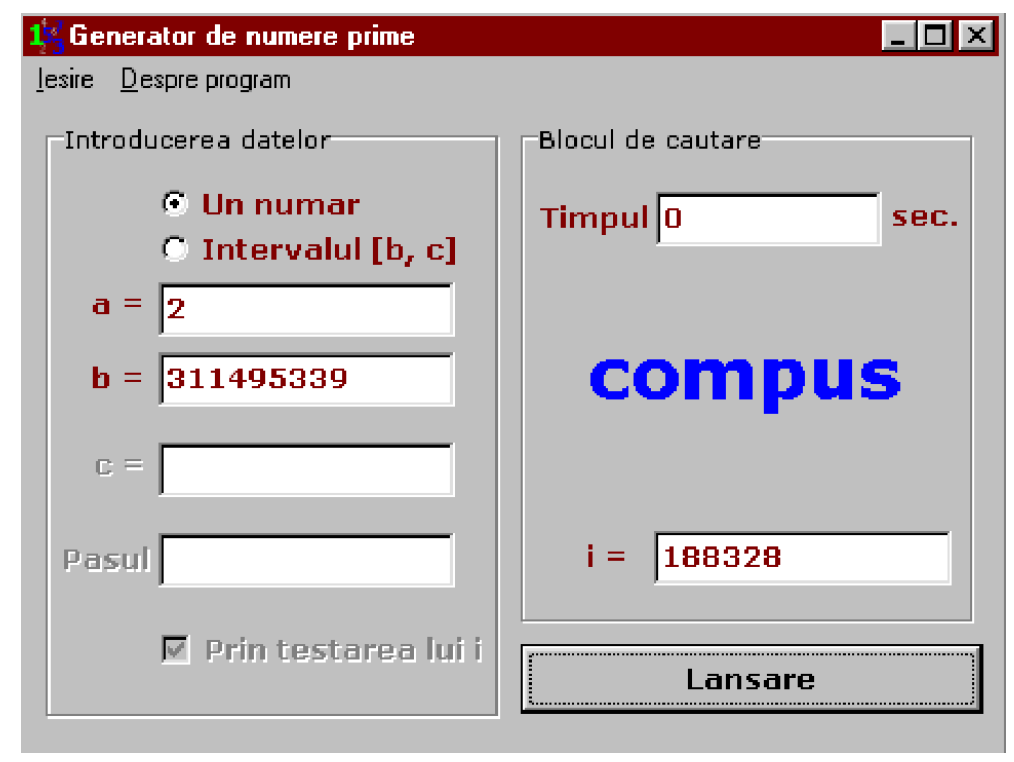

Figura 2: Caso de un número compuesto.

$\mathrm{Al}$ activar el círculo Intervalul [b,c], podemos hallar todos los números primos del intervalo $[\mathrm{b}, \mathrm{c}]$. Los extremos del intervalo se escriben en las celdas "b" y "c". En la celda "Pasul" escribimos el paso (la diferencia entre dos números vecinos). La celda "Prin testarea lui i "se queda no marcada. Este caso está indicado en la Fig. 3.

La investigación comienza con la presión del botón Lansare. El intervalo contiene 10 números primos que están indicados en la lista junto con los valores de $i$.

Con otros programas [8] en la base de las propiedades de las matrices multidimensionales, se pueden construir números primos mucho más grandes. $\mathrm{Al}$ conocer un número primo con $k$ cifras, se construye un número primo con $q>k$ cifras. El mayor número primo que se encontró es el número $n=15418\left(2^{86243}-1\right)+1$ que contiene 25966 cifras significativas.

Veamos un caso particular, precisamente se trata de los números de la forma $2^{p}-1$, donde $p$ es un número primo. Estos números se llaman Números de Mersenne $[4,5,6,8]$. Para unos valores de $p$ estos números pueden ser primos, para otros son compuestos. $\mathrm{Al}$ presente se conocen solamente 43 números primos de Mersenne (http://www.mersenne.org/works.htm). El 43-ésimo número fue descubierto en Diciembre del 2005. Este es el número $2^{30402457}-1$ que contiene 9152052 cifras significativas. Este es el mayor número primo conocido en la ciencia. Todavía no se conoce si el conjunto de los números primos de Mersenne es finito o infinito. Los números compuestos de Mersenne son muchos más. El conjunto de estos números tampoco se conoce si es finito o infinito. El generador de números primos descrito anteriormente, puede servir como generador de números cumpuestos de Mersenne. Para eso activamos el círculo "Intervalul [b,c]" y la celda "Prin testarea lui i", elegimos dos números $b, c \in K_{i}$ tales que $(i, m)=1$ y los escribimos en las celdas "b" y "c" respectivamente. En las celdas "a" y "Pasul" es- 


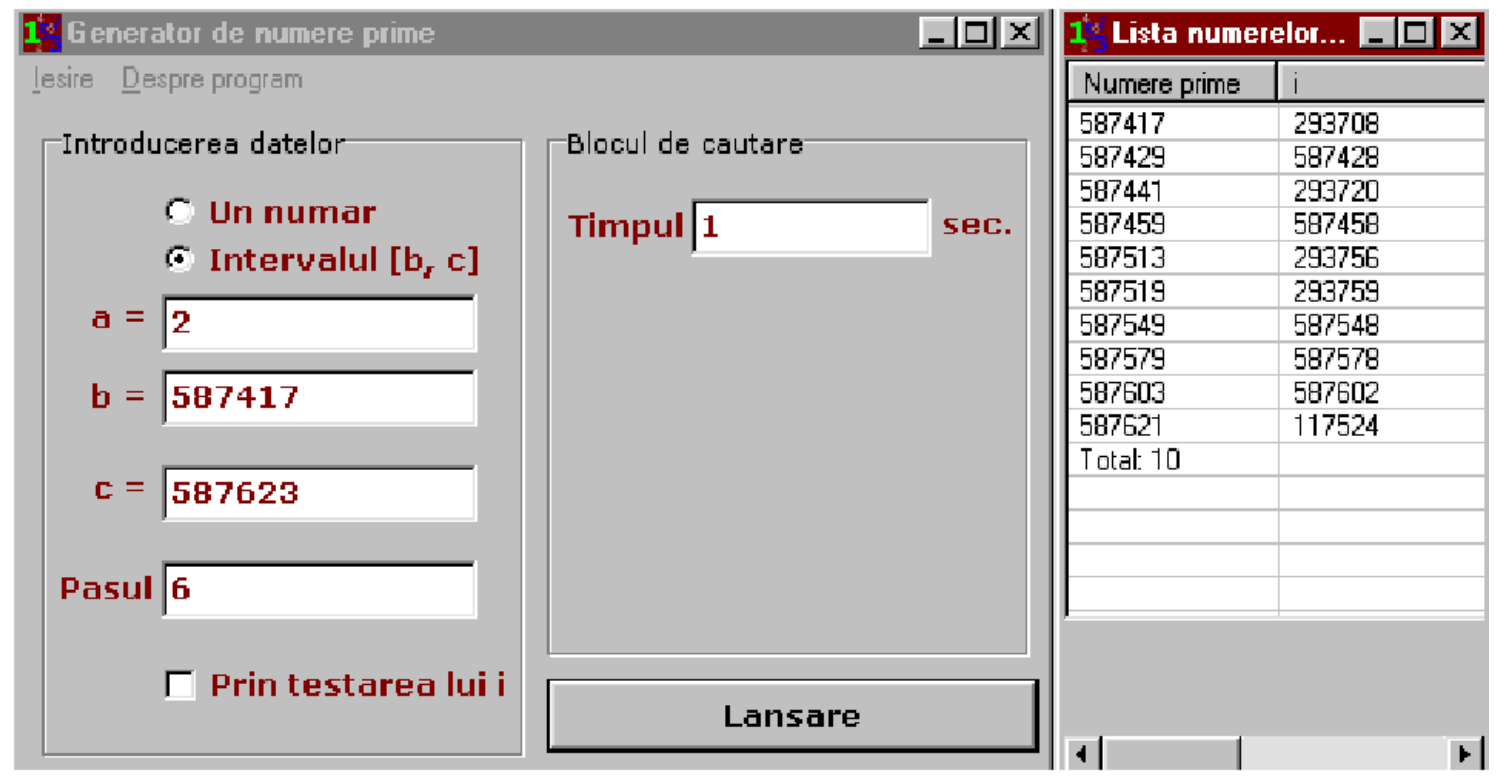

Figura 3: Números primos en el intervalo $[b, c]$.

cribimos 2 y el paso respectivamente. La investigación comienza con la presión del botón "Lansare". El resultado de la investigación es la lista de los valores de $p(p=i)$ en los números compuestos de Mersenne y de los divisores respectivos de estos números. Veamos un caso concreto. Los números 2002098071 y 2139826583 pertenecen a la línea $K_{5}$ de la matriz (8) (se cumple la condición (9)) para $n=12$. Ya que $(5,6)=1$, entonces en la línea $K_{5}$ hay números primos. Según el teorema 1 tomamos $s=2002098071$ y $k=7$. Con estas condiciones los números indicados son términos de una progresión aritmética con el primer término $s$ y con la razón $6^{7}=279936$. Todos los términos de esta progresión pertenecen a la línea $K_{5}$. Marcamos el círculo "Intervalul [b,c]" y la celda "Prin testarea lui i". En las celdas "a", "b" y "c" escribimos respectivamente 2,2002098071 y 2139826583 . El resultado de la investigación está indicado en la Fig. 4.

De esta manera, hemos obtenido cuatro números compuestos de Mersenne: $2^{1001468939}-1,2^{2004968719}-1,2^{1053816971}-1$, y $2^{1061515211}-1$ con sus divisores respectivos 2002937879, 2049687191, 2107633943 y 2123030423 . Tomando otros intervalos se obtuvieron otros números: $2^{5000062311}-1,2^{6124388391}-1,2^{1000000871}-1$ y $2^{1000001759}-1$ con sus divisores respectivos 1000012463, 1224877679, 2000001743 y 2000003519.

Con otros programas [8] se encontraron divisores de números Mersenne compuestos mucho más grandes. El mayor de ellos es el número $2^{p}-1$ donde

$p=37566209334489046488144568092294833051422790541629075043603903519311342796$ 002563317961928227247467514201588721186924893262288479372404158434263818462065 337092970189574337252841546920960399647393608111916071814812203589093130845171 960949886781342697601464780553018463047715973386696326964233482586186690647498 417876722482308474728847448834198727492857350730001814074054395386577331967424 594781286596363864920580662819596012007216922996947448252865591537557767228654 891215414705559111276602641461508931642316984956651285657246192284266086453530 055308127171898542626967064306829748306299659537928642062378119553961547183922 


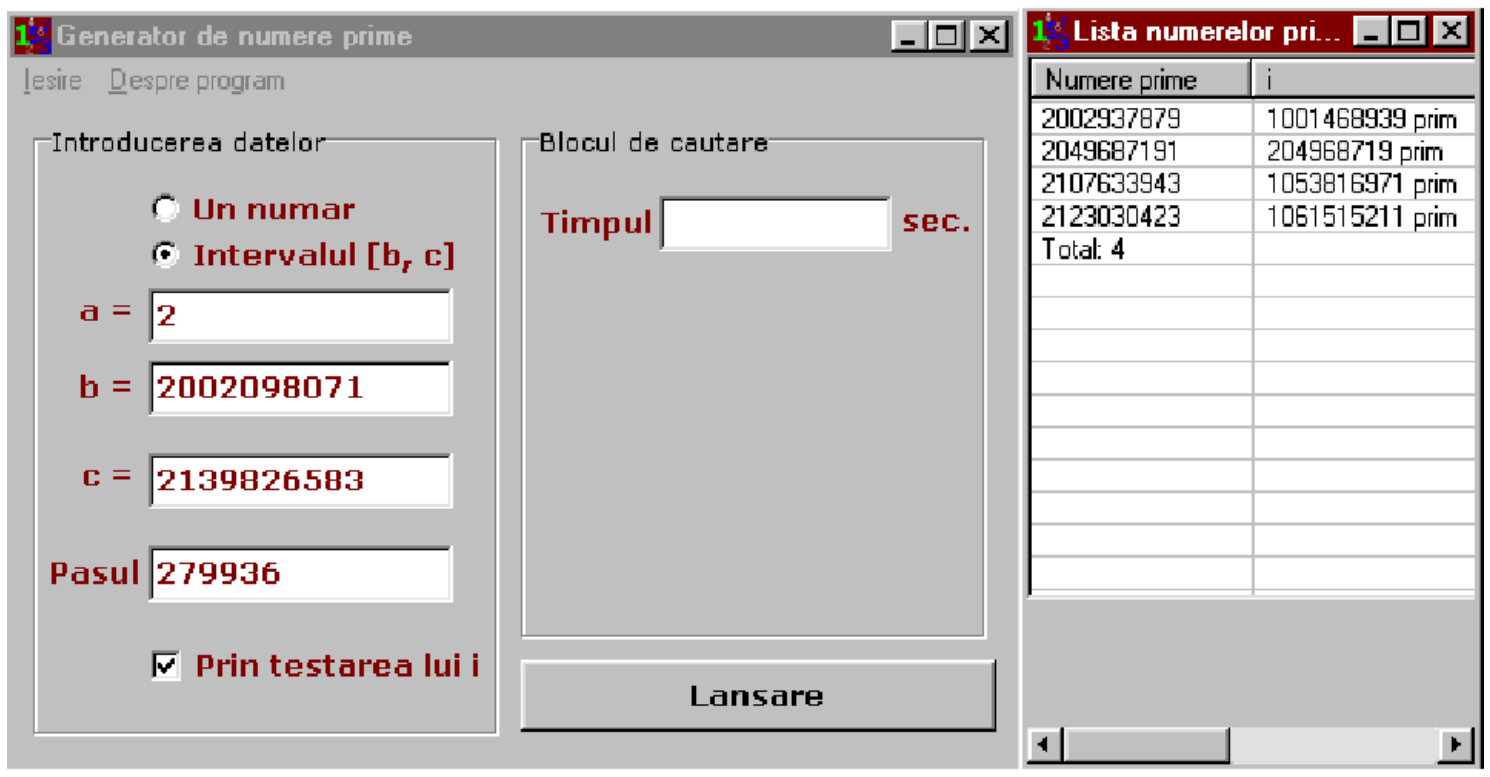

Figura 4: Números compuestos de Mersenne.

917153728040093521102920824572718113115971366333371468967629081531867519214588 952683887819985272623352019398976015727665307773957741164365060083908931250128 185602087151549765139791238422988511958507121831373840715454168939282504348228 725483489795029437636225111813384638621685522611518395287014588528008037325320 351289421909807691457658164032115851278974493764051564096909639254478507218537 346290382647945537653532393918686640643071936605435920613489508262594316606776 3036796988950518528130951459622923007.

Este número tiene el divisor primo:

$d=60105934935182474381031308947671732882276464866606520069766245630898148473$ 604101308739085163595948022722541953899079829219661566995846653494822109539304 539348752303318939604546475073536639435829772979065714903699525742549009352275 137519818850148316162343648884829540876345557418714123142773572137898705035997 468602755971693559566155918134717963988571761168002902518487032618523731147879 351650058554182183872929060511353619211547076795115917204584946460092427565847 825944663528894578042564226338414290627707175930642057051593907654825738325648 088493003475037668203147302890927597290079455260685827299804991286338475494276 667445964864149633764673319316348980985554186133394350348206530450988030743342 324294220511976436197363231038361625164264492438332385862984096134254290000205 096963339442479624223665981476781619133611394930198145144726670302852006957165 960773583672047100217960178901415421794696836178429432459223341644812859720512 562063075055692306332253062451385362046359190022482502555055422807165611549659 754064612236712860245651830269898625028915098568697472981583213220150906570842 08588751823208296450095223353966768113 , que contiene 1126 cifras significativas.

A propósito, todavía no se conoce ningún divisor de los números compuestos $2^{751}-1$, $2^{809}-1,2^{997}-1$, y $2^{1061}-1$. 


\section{Factorizadores de números compuestos}

Veamos la matriz (7). Sea la función de Euler $\varphi(m)=s$. Componemos el conjunto de números $M=\left\{m_{1}, m_{2}, \ldots, m_{s}, m_{s+1}\right\}$, donde $s=\varphi(m), \quad\left(m_{i}, m\right)=1$ y $m_{i}<m$ para $i=1, \ldots, s ; \quad m_{1}=1, m_{s}=m-1$ y $m_{s+1}=m+1$. Componemos el conjunto $\Delta=\left\{\Delta_{1}, \Delta_{2}, \Delta_{3}, \ldots, \Delta_{s}\right\}$ donde $\Delta_{k}=m_{k+1}-m_{k}$ para $k=1, \ldots, s$. Es fácil ver que para cualquier $m$ se cumple

$$
\sum_{k=1}^{s} \Delta_{k}=m
$$

En efecto, $\sum_{k=1}^{s} \Delta_{k}=\Delta_{1}+\Delta_{2}+\Delta_{3} \ldots+\Delta_{s}=m_{2}-m_{1}+m_{3}-m_{2}+m_{4}-m_{3}+\cdots+m_{s+1}-m_{s}=$ $-m_{1}+m_{s+1}=-1+m+1=m$.

Ya que $\left(m_{i}, m\right)=1$, entonces los números primos están en las líneas $K_{m_{1}}, K_{m_{2}}$, $\ldots, K_{m_{i}}, \ldots, K_{m_{s}}$. Construimos la matriz con estas líneas y designamos las columnas por $0,1,2,3, \ldots, m^{n-1}-1$.

$$
\begin{gathered}
K_{m_{1}} \\
K_{m_{2}} \\
\vdots \\
K_{m_{i}} \\
\vdots \\
K_{m_{s}}
\end{gathered}\left[\begin{array}{ccccc}
N_{1} & N_{s+1} & N_{2 s+1} & \ldots & N_{\left(m^{n-1}-1\right) s+1} \\
N_{2} & N_{s+2} & N_{2 s+2} & \ldots & N_{\left(m^{n-1}-1\right) s+2} \\
\vdots & \vdots & \vdots & \ddots & \vdots \\
N_{i} & N_{s+i} & N_{2 s+i} & \ldots & N_{\left(m^{n-1}-1\right) s+i} \\
\vdots & \vdots & \vdots & \ddots & \vdots \\
N_{s} & N_{2 s} & N_{3 s} & \ldots & N_{m^{n-1} s}
\end{array}\right]
$$

En esta matriz $N_{1}=1, N_{2}=N_{1}+\Delta_{1}, N_{3}=N_{2}+\Delta_{2}, \ldots, N_{s}=N_{s-1}+\Delta_{s-1}, N_{s+1}=$ $N_{s}+\Delta_{s}$. Luego $N_{s+2}=N_{s+1}+\Delta_{1}, N_{s+3}=N_{s+2}+\Delta_{2}$ y así sucesivamente. Los divisores de cualquier número natural $N$ están sobre estas líneas. En la base de las propiedades de las matrices multidimensiomales se elaboró un factorizador de números de la forma $a^{i}-1$ para $a=2$. El programa verifica para cuáles valores de $N_{k}$ de la matriz (17) se cumple la condición

$$
2^{i} \equiv 1\left(\bmod N_{k}\right)
$$

En la Fig. 5 está indicado el factorizador que factorizó el número compuesto de Mersenne $2^{71}-1$ cuando $m=2 \cdot 3 \cdot 5 \cdot 7=210$. En la celda "i" se escribe el valor de $i$ de la condición (18).

El programa tiene dos formas de funcionamiento:

(*) La búsqueda se efectúa en todas las líneas de la matriz (17) pasando columna tras columna.

(**) La búsqueda se realiza solamente en una línea concreta.

En Configurare elegimos la forma de funcionamiento. Al activar prime se escoge la forma $(*)$. Otra forma se escoge cuando activamos Pas. Primero se escoge la forma de 


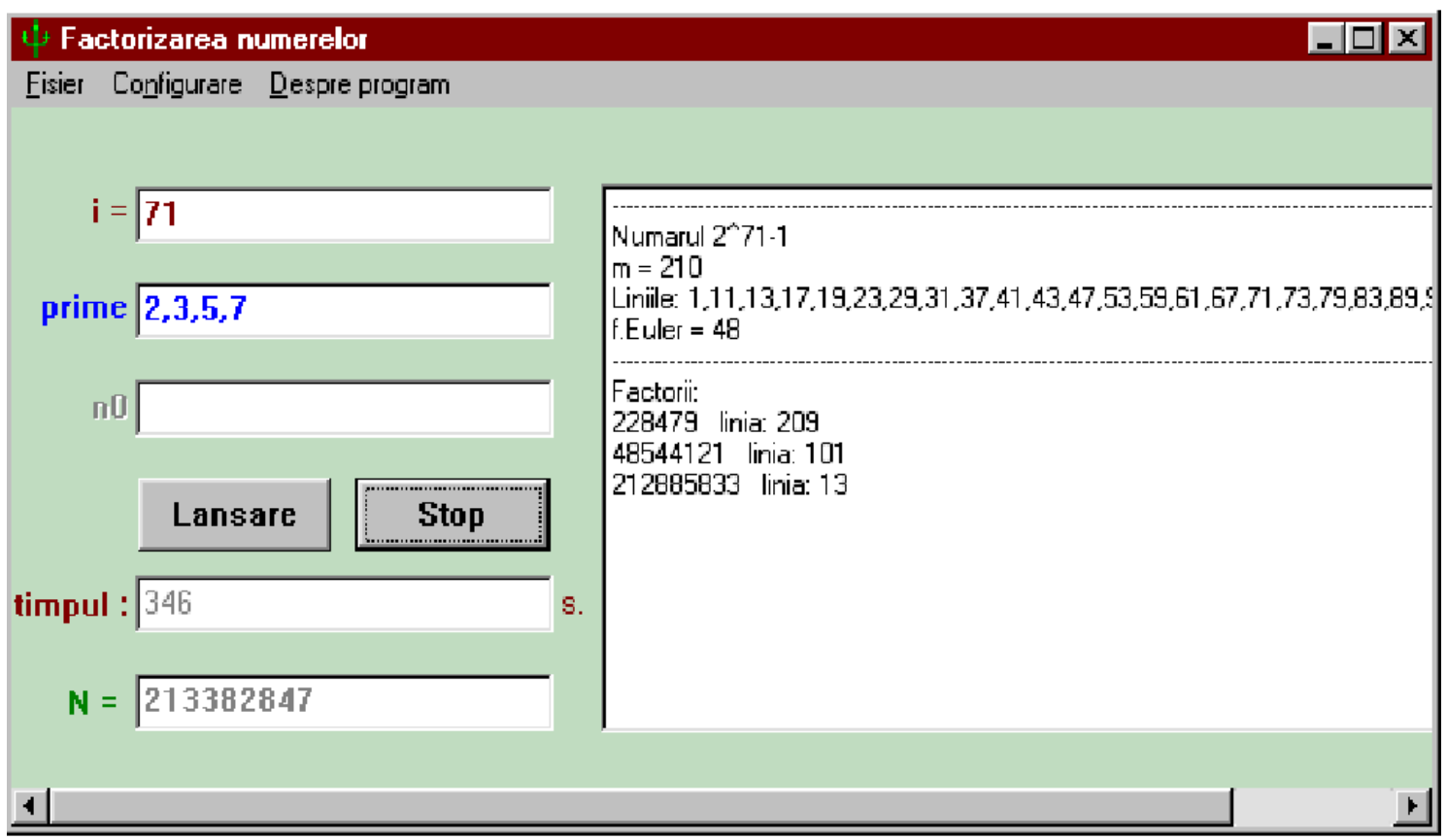

Figura 5: Factorización de $2^{71}-1$ cuando $m=2 \cdot 3 \cdot 5 \cdot 7=210$.

funcionamiento y después se introducen otros datos. En la forma $(*)$ en la celda "Prim", escribimos los factores $p_{i}$ de $m=p_{1} \cdot p_{2} \cdot \ldots \cdot p_{c} ; c \in\{1,2,3,4\}$ y $p_{i} \in\{2,3,5,7\}$. Al activar Lansare en la pantalla aparece el número que se factoriza, el valor de $m$, los valores de $m_{i} \mathrm{y}$ la función de Euler $s=\varphi(m)$. Más abajo, después de la investigación, aparecen los factores de $N$ y las líneas de la matriz (17), sobre las cuales se encuentran los factores respectivos. En la celda timpul se indica la duración de la investigación. En la celda "N" aparece el último número que se investigó. Si deseamos saber cuál número se está investigando en cualquier momento, activamos Stop. El número aparece en la celda $N$. Para continuar activamos Continuati. De la Fig. 5 tenemos: $N=2^{71}-1=2361183241434822606847=$ $228479 \cdot 48544121 \cdot 212885833$.

Hace falta señalar que en la lista de los factores aparecen tanto los divisores primos, como unos de los divisores compuestos del número $N$. Si es necesario sacamos de la lista los factores primos.

Si quisiéramos guardar el resultado de la investigación, escogemos Nou en Fisier. Lo mismo hacemos cuando pasamos a la factorización de otro número. En la Fig. 6 está indicado el resultado guardado de la factorización del número $2^{71}-1$.

$\mathrm{Al}$ escoger la forma (**), en la celda pas escribimos el valor de $m$ y en la celda $n_{0}-$ el número $m_{i}$ de la línea que se examina en la matriz (17).

Hace falta señalar que los factores $p_{i}$ del número $m$, pueden ser divisores del número $N$ que se factoriza. Estos divisores no salen en la lista. La existencia de estos divisores se verifica aparte.

El factorizador funciona con las restricciones:

$$
2 \leq i \leq 2200000000, \quad 2 \leq N_{k} \leq 1100000001 .
$$

La rapidez de la búsqueda de los divisores del número $a^{i}-1$ depende considerablemente de la elección de $m$. Este problema requiere una investigación aparte más profunda. Por 


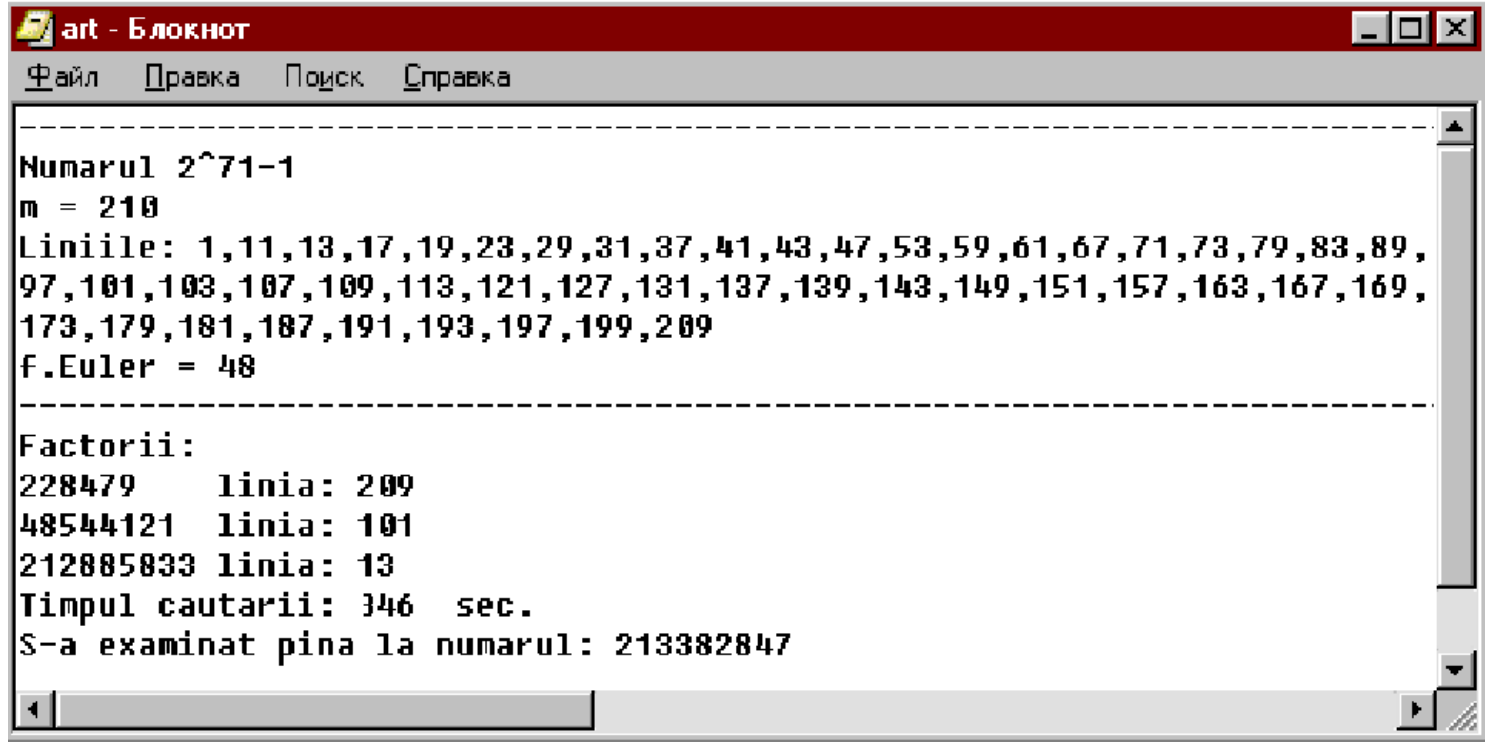

Figura 6: Factorización del número $2^{71}-1$.

el momento mencionemos que en la forma de funcionamiento $(*), m$ debe tener el mayor valor posible. En el factorizador elaborado este valor es igual a 210. Pero la variante óptima se obtiene en la forma de funcionamieto $(* *)$ cuando

$$
n_{0}=1 \quad \text { y } \quad m=d \cdot i
$$

donde $d$ es el máximo común divisor de los números $\frac{p_{1}-1}{i}, \frac{p_{2}-1}{i}, \ldots, \frac{p_{k}-1}{i}$; $p_{1}, p_{2}, \ldots, p_{k}$ son todos los divisores primos del número $a^{i}-1$. La factorización del número $2^{71}-1$ con las condiciones (20) (para $d=2, i=71$ ) está indicada en la figura 7 . La factorización se realizó mucho más rápidamente que en el caso cuando $m=210$.

En la forma de funcionamiento $(*)$, no siempre salen los divisores menores que $i$ y a veces tampoco salen unos divisores mayores que $i$. Estos divisores se buscan adicionalmente.

En todos los ejemplos indicados, las investigaciones se hicieron con una computadora PII-400MHz.

\section{Conclusiones}

1. Los generadores y factorizadores elaborados son compactos y cómodos para la utilización. Los usuarios no necesitan conocimientos especiales.

2. Los factorizadores son elaborados solamente para números especiales de la forma $a^{i}-1$ para $a=2$. Es fácil transformar los programas para $a$ arbitrario. Para otras formas se necesitan otros programas usando las mismas propiedades de las matrices multidimensionales. 


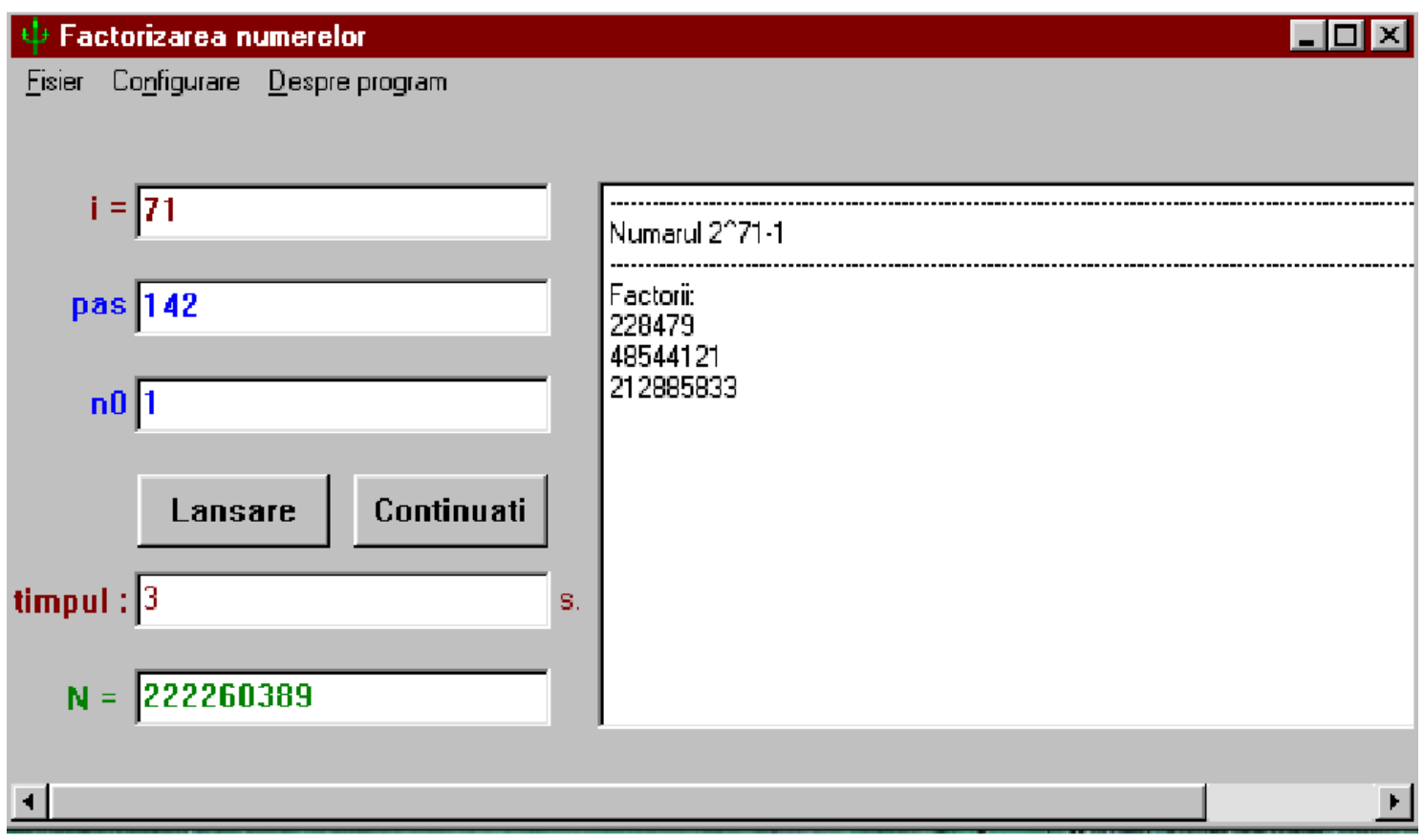

Figura 7: La factorización del número $2^{71}-1$ con las condiciones (20).

3. Los intervalos de las restricciones (15) y (19) pueden ser ensanchados prácticamente de un modo ilimitado. Para eso se puede, por ejemplo, pasar a la otra forma de representación de los números. Los números pueden representarse como polinomios $P_{n}(m)=a_{0}+a_{1} m+a_{2} m^{2}+\ldots+a_{n} m^{n}$ de potencias de $m$. Teniendo en cuenta la estructura de la matriz (17) y la condición (16), es fácil obtener los elementos de esta matriz en forma de polinomio.

4. El factorizador de números puede servir también como generador de números primos, ya que los factores que se buscan son números primos. Al factorizar los números compuestos de Mersenne, podemos obtener números primos gigantescos en la forma polinomial. Estos números se pueden hallar investigando los elementos de la línea $K_{1}$ cuando $m=d \cdot i$.

5. Para buscar los números primos de Mersenne sería bueno elaborar una red de computadores que investiguen (en la forma $(* *)$ de funcionamieto) todas las líneas de la matriz (17).

\section{Referencias}

[1] Bulat, M. (2001) "Isomorfismo de grandes sistemas", Acta Academia 2001, Evrica, Chisinau: $161-170$.

[2] Bulat, M. (2000) "Isomorphic sistems of graphs", $3^{\text {rd }}$ European Congress of Mathematics, Section 06: Discrete Mathematics and Computer Science, Poster number 236, Barcelona. 
[3] Bulat, M. (2002) "Algunas aplicaciones de las matrices multidimensionales", Anales ATIC-2002, vol.1, Academia de Transportes, Informática y Comunicaciones, Chisinau, Evrica: 30-38 (en rumano).

[4] Bulat,M. (2003) "La distribución de los números primos en matrices multidimensionales", Anales ATIC-2003, vol.1, Academia de Transportes, Informática y Comunicaciones, Chisinau, Evrica: 74-82 (en rumano).

[5] Minuts, P. (1997) Teoria de los Números, Vol.1, Editorial Crengutsa Galdau, Iasi (en rumano).

[6] Oleinik, W.(1999) "Métodos de obtención de los números primos. La situación actual y las perspectivas." Acta Academia 1999, Evrica, Chisinau: 101-126 (en ruso).

[7] Bajandin, E.(2003) La distribución de los Números Primos en la Serie de los Números Naturales. Editorial Nauka, Novosibirsk (en ruso).

[8] Bulat, M.; Zgureanu, A.; Ciobanu, I.; Bivol, L. (2006) "A method for obtaining arbitrary form prime numbers", Satellite Conference of the ICM 2006. The XIV ${ }^{t} h$ Conference on Applied and Industrial Mathematics, Chisinau: 70-73. 